\title{
Sexuelle Dysfunktionen bei Patienten einer verhaltenstherapeutischen Hochschulambulanz: Häufigkeit, Erkennen, Behandlung
}

\author{
Andrea Reinecke Daniel Schöps Jürgen Hoyer \\ Klinische Psychologie und Psychotherapie, Technische Universität Dresden, Deutschland
}

\section{Schlüsselwörter}

Sexualität · Sexuelle Dysfunktion - Sexuelle Funktionsstörung · Psychotherapeutische Versorgung · Ausbildung

\section{Zusammenfassung}

Hintergrund: Prävalenzdaten weisen auf einen hohen therapeutischen Versorgungsbedarf für sexuelle Dysfunktionen hin. Unser Ziel war es zu untersuchen, inwieweit ein solcher Bedarf bei Patienten einer psychotherapeutischen Hochschulambulanz besteht und inwieweit die sexuellen Probleme von vorwiegend in Ausbildung befindlichen Verhaltenstherapeuten erkannt und therapeutisch aufgegriffen werden. Patienten und Methoden: In einer Patientenstudie (Studie 1) wurden 173 Psychotherapiepatienten (16-64 Jahre, 71,7\% Frauen) mittels des Kurzfragebogens Sexualität und Partnerschaft (KFSP) zu ihrem sexuellen Interesse, ihrer sexuellen Erregungsfähigkeit, ihrer Orgasmusfähigkeit, ihrer Erektionsbzw. Lubrikationsfähigkeit sowie ihrer sexuellen Zufriedenheit im letzten Monat befragt. In der Therapeutenstudie (Studie 2) wurde untersucht, ob 16 vorwiegend in Ausbildung befindliche Therapeuten Patienten mit bzw. ohne sexuelle Dysfunktion als solche erkennen und die Probleme thematisieren und behandeln. Ergebnisse: Je nach Problembereich äußert jede zweite bis dritte Frau bzw. jeder dritte bis fünfte Mann sexuelle Probleme. Therapeuten erkennen sexuelle Schwierigkeiten bei jedem zweiten Betroffenen. Sexualität wird bei jedem zweiten Patienten thematisiert. Jede dritte richtig erkannte Dysfunktion wird auch behandelt. Schlussfolgerung: Erkennen und Behandeln sexueller Dysfunktionen sollten im Rahmen der Ausbildung stärker berücksichtigt werden.

\section{Key Words}

Sexuality - Sexual dysfunction . Psychotherapeutic care . Behavioural therapy training

\section{Summary}

Sexual Dysfunctions in Patients of a CBT University Outpatient Clinic: Frequency, Recognition, and Treatment Background: Prevalence data on sexual dysfunctions indicate a high need for therapy and health care for sexual problems. One of the study's aims was to investigate the extent of that need in patients of a psychotherapeutic university outpatient clinic. Besides, we examined to what extent sexual problems are recognised and treated by behaviour therapists in training. Patients and Methods: In a patient study, we tested 173 outpatients (aged 16-64 years, $71.7 \%$ female) who were seeking psychotherapy. By completing the German version of the Massachusetts General Hospital Sexual Functioning Questionnaire, participants rated their sexual interest, their ability to sexual arousal, to experience orgasm, to attain erection/lubrication and their general sexual satisfaction in the past month. In a therapist study, we examined whether 16 therapists in training were able to differentiate between patients with and without sexual dysfunction, whether they brought up the topic during therapy and whether they treated the sexual dysfunction. Results: Depending on the type of problem, one out of two to three women and one out of three to five men report sexual problems. Therapists recognise sexual problems in half of the patients, and bring up the issue of sexuality in every second patient. In fact, every third case of sexual dysfunction is treated. Conclusion: Behaviour therapy training should put a stronger emphasis on the topic of 'sexual dysfunctions'.

\begin{tabular}{ll}
\hline KARGER & @ 2006 S. Karger GmbH, Freiburg \\
$\begin{array}{l}\text { Fax +49 761 4520714 } \\
\begin{array}{l}\text { E-mail Information@Karger.de } \\
\text { www.karger.com }\end{array}\end{array}$ & $\begin{array}{c}\text { Accessible online at: } \\
\text { www.karger.com/ver }\end{array}$
\end{tabular}




\section{Hintergrund}

Der Umgang mit dem Thema Sexualität ist heute sicherlich aufgeklärter als früher. Es scheint aber, dass ein offensiver dabei oft provokativer - Umgang den Medien vorbehalten bleibt. Sexuelle Probleme sind und bleiben ein persönliches und intimes Thema. Dies könnte dazu führen, dass sie trotz gegebener Behandlungsmöglichkeiten nicht erkannt und nicht adäquat versorgt werden. Dazu passt die Beobachtung, dass Sexualität und sexuelle Dysfunktionen in der verhaltenstherapeutischen Ausbildung meist eine untergeordnete Rolle spielen. Dabei weisen epidemiologische Daten zu sexuellen Problemen auf einen hohen therapeutischen Bedarf hin.

Einer Studie von Laumann et al. [1999] zufolge leiden 43\% der Frauen und 31\% der Männer (12-Monats-Prävalenz) der US-amerikanischen Allgemeinbevölkerung im weitesten Sinne an sexuellen Problemen: etwa jede dritte Frau (32\%) klagt über einen Verlust oder Mangel an sexuellem Verlangen, etwa jede vierte Frau über Orgasmusstörungen (26\%), sexuelle Aversion bzw. mangelnde sexuelle Befriedigung $(23 \%)$, jede fünfte über Erregungs- bzw. Lubrikationsstörungen $(21 \%)$ und jede sechste über Dyspareunie (16\%). Ähnlich hohe Angaben zur 12-Monats-Prävalenz finden sich bei Nicolosi et al. [2004]. In geringerem Ausmaß werden sexuelle Beeinträchtigungen bei Männern berichtet. Laumann et al. [1999] zufolge leiden 5\% der Männer der Allgemeinbevölkerung an einem verloren gegangenen oder reduzierten sexuellen Verlangen; jeder fünfte an vorzeitiger Ejakulation (21\%). Nicolosi et al. [2004] schätzen, dass 28\% der Männer sexuelle Probleme haben, wobei vorzeitige Ejakulation (14\%) und erektile Dysfunktion (10\%) am häufigsten angegeben werden.

Noch höhere Prävalenzangaben finden sich für klinische Gruppen. 41\% der Frauen in stationärer psychotherapeutischer Behandlung geben einen Verlust oder Mangel an sexuellem Verlangen an. Bonierbale et al. [2003] berichten gar eine Prävalenz sexueller Dysfunktionen unter depressiven Frauen von $69 \%$. Untersuchungen an alkoholabhängigen [Fahrner, 1985], psychosomatischen [Kommer et al., 1990] oder psychiatrischen [Kockott und Pfeiffer, 1996] männlichen Patienten zufolge weisen etwa $50 \%$ davon behandlungsbedürftige Sexualstörungen auf. Labbate und Lare [2001] fanden sogar noch höhere Raten (62-67\%). Ferner sind enge Assoziationen sexueller Beeinträchtigungen mit Angst, Depression und Essstörungen [Ernst et al., 1993] bzw. Alkoholmissbrauch [Johnson et al., 2004; O Farrell et al., 1997] belegt.

Zunehmend werden die in der Literatur berichteten Prävalenzen sexueller Störungen jedoch als überzogen in Frage gestellt. Einerseits erfassen Studien oft lediglich sexuelle Probleme statt klassifizierbarer Diagnosen. Sexuelle Dysfunktionen spiegeln Probleme mit Verlangen, Erregung und Orgasmus sowie Schmerzen während sexueller Aktivität wider. Sexuelle Funktionsstörungen (ICD-10 F52) hingegen sind nur zu diagnostizieren, wenn Dysfunktionen auch zu interpersonellen Beeinträchtigungen führen und nicht auf eine andere Achse-I-
Tab. 1. Items des Kurzfragebogens Sexualität und Partnerschaft [Labbate und Lare, 2001; Hoyer, 2003]. Die Beantwortung erfolgt durch ein 6-stufiges Rating von «größer als sonst» bis «nicht vorhanden»

\section{Nr. Item}

Wie war Ihr sexuelles Interesse im letzten Monat?

Wie war Ihre sexuelle Erregungsfähigkeit im letzten Monat?

Wie war Ihre Orgasmusfähigkeit im letzten Monat?

(Nur für Frauen) Wie war Ihre Lubrikation im letzten Monat? (Nur für Männer) Wie war Ihre Erektionsfähigkeit im letzten Monat?

5 Wie schätzen Sie insgesamt Ihre sexuelle Zufriedenheit im letzten Monat ein?

$1=$ größer als sonst, $2=$ normal, $3=$ minimal geringer als sonst, $4=$ etwas geringer als sonst, $5=$ bedeutend geringer als sonst, $6=$ nicht vorhanden .

Störung, das Alter, medizinische Faktoren oder Medikamente zurückgehen. Andererseits wird nicht in allen Studien das $\mathrm{Zu}$ satzkriterium «subjektiver Leidensdruck» (bei Dunn et al. [1998] z.B. operationalisiert als Äußerung von Behandlungsbedarf) berücksichtigt. Zusätzlich verwenden verschiedene Studien unterschiedliche Messinstrumente. Moynihan [2003] vermutet darüber hinaus, dass profitorientierte Pharmakonzerne Bemühungen unterstützen, neue, pharmakologisch behandlungswürdige Diagnosen sexueller Funktionsstörungen zu definieren.

Selbst wenn einige Studien die Prävalenz sexueller Dysfunktionen überschätzen sollten, kann aufgrund therapeutischer Erfahrung angenommen werden, dass sexuelle Probleme häufig vorkommen und von subjektivem Leidensdruck begleitet sind. Wie aber sieht die Realität der Versorgung sexueller Probleme aus? Dunn et al. [1998] erfassten nicht nur Prävalenzschätzungen verschiedener sexueller Probleme in der Allgemeinbevölkerung, sondern auch, wie häufig deren Behandlung gewünscht und angeboten wird. Demnach äußerte jeder zweite Proband mit sexuellen Problemen auch einen Wunsch nach Behandlung, aber nur jeder Zehnte erhielt tatsächlich Hilfe. Das Missverhältnis zwischen notwendiger und realer Versorgung unterstreicht eine Untersuchung zur Behandlung des Themas Sexualität in Fertilitätskliniken [Meerabeau, 1999], wonach in nur 6 von 160 Konsultationen offen über Sexualität gesprochen wurde. Andererseits sind Patienten oft zu Gesprächen bereit, daher könnte mehr Initiative von Seiten des Arztes zu größeren Versorgungserfolgen führen [z.B. Bonierbale et al., 2003; Nusbaum et al., 2004; Read et al., 1997]. Besonders ältere Patientinnen wünschen sich, vom Arzt auf ihre sexuelle Gesundheit angesprochen zu werden [Nusbaum et al., 2004]. Ähnliche Ergebnisse finden sich bei Bonierbale et al. [2003], die die Prävalenz sexueller Dysfunktionen unter mehr als 4500 depressiven Frauen untersuchten. Nur jede dritte Frau äußerte sexuelle Beeinträchtigungen spontan, auf Nachfragen durch den Arzt waren es jedoch 69\%.

Psychotherapeuten würde man unterstellen, dass sie besonders kompetent sein sollten schwierige und heikle Themen 
wie sexuelle Dysfunktionen aufzugreifen und anzusprechen. Oder spielt die «Vermeidung» der Sexualität auch in der verhaltenstherapeutischen Behandlungspraxis eine Rolle? Daten zu diesen Fragen fehlen weitgehend. Im Folgenden stellen wir deshalb eine Patientenstudie (Studie 1) und eine Therapeutenstudie (Studie 2) vor, die die Häufigkeit sexueller Dysfunktionen in der therapeutischen Praxis sowie das Ausmaß des Erkennens und Behandelns sexueller Dysfunktionen durch Therapeuten untersucht haben.

\section{Patienten und Methoden}

\section{Patientenstudie}

\section{Stichprobe}

Untersucht wurden 173 ambulante Psychotherapiepatientinnen und -patienten der Institutsambulanz für Psychotherapie der Technischen Universität Dresden. 71,7\% der Probanden waren weiblich. Die Teilnehmer waren 16-64 Jahre alt (Mittelwert $M=34,5$ ). 95,2\% gaben an, heterosexuell orientiert zu sein, jeweils $2,4 \%$ waren homosexuell bzw. bisexuell orientiert. Alle Patienten wurden mit dem Composite International Diagnostic Interview der WHO [deutsche, modifizierte Version: M-CIDI, Wittchen und Pfister, 1997] diagnostiziert. Im klinischen Gesamturteil erfüllten $65,1 \%$ die ICD-10-Kriterien für irgendeine Angststörung und $50,5 \%$ die Kriterien für eine affektive Störung. In wesentlich geringerer Häufigkeit wies die Patientengruppe Persönlichkeits- und Verhaltensstörungen $(6,4 \%)$, Verhaltensauffälligkeiten mit körperlichen Störungen oder Faktoren $(5,5 \%)$, Störungen durch psychotrope Substanzen und wahnhafte Störungen (je 1,8\%) sowie Verhaltens- und emotionale Störungen mit Beginn in der Kindheit und Jugend (0,9\%) auf.

\section{Screening-Instrument}

Patienten, die nach Aufklärung über die Untersuchung der Teilnahme zustimmten, füllten während der routinemäßigen Eingangsuntersuchung der Ambulanz den «Kurzfragebogen Sexualität und Partnerschaft» [KFSP; Labbate und Lare, 2001] aus (Tab. 1). Dieser Fragebogen verfolgt eine reine Screening-Zielsetzung, so dass die herkömmlichen Testgütekriterien nur eingeschränkt anwendbar sind [Hoyer et al., 2003]. Dieses international bewährte Instrument ist zwar sehr zeitökonomisch, seine Augenscheinvalidität ist jedoch eingeschränkt. So bleibt zum einen der individuelle Leidensdruck der Probanden unberücksichtigt, zum anderen bleibt unklar, hinsichtlich welchen Vergleichszeitraums die Antwortkategorie «normal» gewählt wurde. Nach längeren Störungsverläufen kann es zum Beispiel «normal» sein, eine eingeschränkte Erregungsfähigkeit zu haben, so dass Unterschätzungen der Prävalenz von Dysfunktionen möglich sind. Auch wird nicht gefragt, inwieweit organische oder altersbedingte Ursachen bekannt sind.

Wir haben die prädiktive Validität des Instruments deshalb in einer begleitenden Studie untersucht [Reitz et al., 2005] und überprüft, inwieweit ein positives Screeningresultat im KFSP mit einer Diagnose einer sexuellen Funktionsstörung nach einem ausführlichen, von Experten durchgeführten strukturierten Interview übereinstimmt [Strukturiertes Interview sexueller Funktionsstörungen, SISEX; Reitz et al., 2005]. Eine Analyse der Übereinstimmung zwischen durch den KFSP bzw. durch das SISEX als «gesund» vs. «Indexpatient» klassifizierten Probanden von $71 \%$ ( $\kappa=$ $0,37, \mathrm{p}=0,013$ ) deutet darauf hin, dass das von uns gewählte Zugangsinstrumentarium trotz seiner Kürze und inhaltlicher Kritikpunkte erstaunlich zuverlässig ist.

Nachfolgende ROC-Analysen der Ergebnisse von Probanden, die sowohl mit Hilfe des KFSP als auch mittels SISEX untersucht wurden, haben ergeben, dass optimale Sensitivität (Proband wird laut KFSP als Indexpa- tient eingestuft und ist tatsächlich einer laut SISEX) und Spezifität (Proband wird laut KFSP als gesund eingestuft, ist dies auch nach SISEX) bei einem KFSP-Trennwert von 3,4 gegeben sind. Mit dem von uns pragmatisch ausgewählten, etwas höher liegenden Trennwert erzielen wir eine Spezifität von 0,909 sowie eine Sensitivität von 0,438. Demnach erfolgt die Zuordnung in die Gruppe «Verdacht auf sexuelle Dysfunktion» mittels der von uns gewählten Trennung auf einer strengeren Basis. Wir können vermuten, dass die Prävalenz sexueller Dysfunktionen etwas höher liegt, als wir sie mittels KFSP ermitteln. Eine Überschätzung ist jedoch nicht zu erwarten.

\section{Therapeutenstudie}

\section{Stichprobe}

In der Therapeutenstudie wurde an insgesamt 16 Therapeuten (davon 12 in Ausbildung) untersucht, inwieweit sie sexuelle Dysfunktionen ihrer Patienten erkennen und thematisieren. Die Therapeuten waren im Durchschnitt $\mathrm{M}=32$ Jahre alt und hatten im Mittel 4,1 Jahre Berufserfahrung als verhaltenstherapeutisch orientierter Psychotherapeut. Die Zusammenstellung 30 parallelisierter Patientenpaare der beiden Gruppen «keine sexuelle Dysfunktion» vs. «Verdacht auf sexuelle Dysfunktion» erfolgte unter pragmatischen Gesichtspunkten auf Grundlage der KFSPWerte. Patienten ohne sexuelle Dysfunktion wurden aus dem Scorebereich <2,6 ausgewählt (das entspricht einem Wert zwischen «normal» und «minimal geringer» bzw. einem Perzentilwert von 46,4), Indexpatienten hatten einen Punktwert >4,75 (das entspricht einem Wert zwischen «etwas geringer» und «deutlich geringer» bzw. einem Perzentilwert von 77,6). Die Patientenpaare waren parallelisiert hinsichtlich Therapeut, Geschlecht und Alter, wobei manchen Therapeuten mehrere Patientenpaare zugeordnet waren. 10 Patientenpaare hatten einen männlichen, 20 Patientenpaare hatten einen weiblichen Therapeuten.

\section{Vorgehen}

Die Behandler wurden hinsichtlich je eines eigenen störungsfreien $\mathrm{Pa}$ tienten und eines Indexpatienten (mit Verdacht auf sexuelle Dysfunktion) aus oben beschriebener Stichprobe befragt. Zu jedem Patienten sollten sie einen einseitigen Fragebogen ausfüllen. Zu den Angaben gehörte eine Einschätzung des Behandlers, ob beim Patienten eine sexuelle Funktionsstörung vorliegt oder nicht, ob diese gegebenenfalls auf eine andere Achse-I-Störung, das Alter oder Medikation zurückzuführen ist bzw. um welche Funktionsstörung es sich handelt. Darüber hinaus erfasste der Fragebogen, ob Therapeuten unterstützend zur Diagnostik sexueller Funktionsstörungen den Eingangsfragebogen eingesehen hatten, der sich ohne Auswertungs- bzw. Interpretationshinweise in der Akte befand. Zusätzlich erfragten wir, ob Therapeuten das Vorliegen sexueller Dysfunktionen in der Therapie explorierten bzw. diese gegebenenfalls behandelten und inwiefern Patienten selbst das Thema Sexualität ansprachen. Ergänzend ermittelten wir die Einschätzung der eigenen Kompetenz zum Behandeln sexueller Dysfunktionen bei Männern und Frauen (5-stufig von «gar nicht» bis «sehr») und der allgemeinen Bedeutung einer sexuellen Dysfunktion für die Behandlung anderer Störungen (5-stufig von «gar nicht» bis «sehr»). In einem zusätzlichen Freitextfeld konnten Gründe für die Nichtbehandlung einer sexuellen Funktionsstörung angegeben werden.

\section{Ergebnisse}

\section{Patientenstudie}

Der Anteil der Patienten, deren Gesamtwerte durchschnittlich im Wertebereich «etwas geringer» (und darunter) liegt, beträgt 32,2\%. Die detaillierten Ergebnisse der Patientenbe- 
Tab. 2. Häufigkeit (in \%) der Wahl einer bestimmten Antwortkategorie für die einzelnen Items, getrennt nach Geschlecht (im oberen Ergebnisteil ist dargestellt, wie viele der 173 Probanden auf ein bestimmtes Item geantwortet haben)

\begin{tabular}{llrlrcc}
\hline Item & & $\begin{array}{c}\text { Sexuelles } \\
\text { Interesse? }\end{array}$ & $\begin{array}{l}\text { Sexuelle } \\
\text { Erregungs- } \\
\text { fähigkeit? }\end{array}$ & $\begin{array}{l}\text { Orgasmus- } \\
\text { fähigkeit? }\end{array}$ & $\begin{array}{l}\text { Erektions-I } \\
\text { Lubrikations- } \\
\text { fähigkeit? }\end{array}$ & $\begin{array}{l}\text { Sexuelle } \\
\text { Zufriedenheit } \\
\text { insgesamt? }\end{array}$ \\
\hline N & Frauen & 124 & 123 & 124 & 71 & 123 \\
& Männer & 49 & 49 & 47 & 52 & 48 \\
& Gesamt & 173 & 172 & 171 & 123 & 171 \\
\hline Größer & Frauen & 5,6 & 4,9 & 2,4 & 2,8 & 3,3 \\
& Männer & 0,0 & 0,0 & 0,0 & 0,0 & 0,0 \\
Normal & Frauen & 36,3 & 42,2 & 46,0 & 60,6 & 37,4 \\
& Männer & 61,2 & 55,1 & 68,1 & 69,2 & 47,9 \\
Minimal & Frauen & 3,2 & 3,2 & 6,5 & 4,2 & 6,5 \\
geringer & Männer & 12,2 & 22,4 & 6,4 & 7,7 & 10,4 \\
Etwas & Frauen & 14,5 & 13,8 & 5,6 & 7,0 & 15,4 \\
geringer & Männer & 10,2 & 8,2 & 14,9 & 9,6 & 14,6 \\
Deutlich & Frauen & 19,4 & 14,6 & 8,1 & 15,5 & 13,8 \\
geringer & Männer & 8,2 & 10,2 & 4,3 & 11,5 & 10,4 \\
Nicht vor- & Frauen & 21,0 & 21,1 & 31,5 & 9,9 & 23,6 \\
handen & Männer & 6,1 & 4,1 & 6,4 & 1,9 & 16,7 \\
\hline
\end{tabular}

fragung sind in Tabelle 2 dargestellt. Unter der Vorannahme, dass Angaben von «minimal geringer» bis «größer» normale Schwankungen sexuellen Interesses und sexueller Funktionen widerspiegeln und Angaben von «etwas geringer» bis «nicht vorhanden» auf Beeinträchtigungen hindeuten, finden wir ähnlich hohe Prävalenzschätzungen wie oben angegeben. Jede zweite Frau (54,9\%) und jeder vierte Mann (24,5\%) berichten demnach ein reduziertes sexuelles Interesse innerhalb der letzten 4-5 Wochen. Jede fünfte Frau gibt sogar an, im letzten Monat gar kein sexuelles Interesse verspürt zu haben. Ebenfalls jede zweite Frau (49,5\%) bzw. jeder vierte bis fünfte Mann (22,5\%) berichtet, sexuell weniger oder gar nicht erregungsfähig gewesen zu sein. Letzteres betrifft jede fünfte Frau der Stichprobe. Reduzierte Orgasmusfähigkeit im letzten Monat beklagen fast jede zweite Frau $(45,2 \%)$ und jeder vierte Mann (25,6\%), wobei fast jede dritte Frau gar nicht zum Orgasmus fähig war. Bei fast jedem vierten Mann (23,0\%) war die Erektionsfähigkeit eingeschränkt, jedoch nur bei einem von 52 gar nicht vorhanden. Fast jede dritte Frau $(32,4 \%)$ berichtet dagegen Probleme in der Lubrikationsfähigkeit, bei jeder zehnten Frau bestand diese Fähigkeit im letzten Monat gar nicht. In der Einschätzung der gesamten sexuellen Zufriedenheit im letzten Monat äußert jede zweite Frau (52,8\%) und jeder zweite bis dritte Mann (41,7\%) eine Verringerung. Fast jede vierte Frau und jeder sechste Mann geben darüber hinaus an, im letzten Monat überhaupt nicht mit ihrer Sexualität zufrieden gewesen zu sein.

Depressionspatienten unterscheiden sich von anderen Patienten in keiner Frage des Screenings signifikant $(\mathrm{t}(107)=0,01, \mathrm{p}$ = 0,997 für den Summenscore des Fragebogens, Alter und Geschlecht kontrolliert). Patienten, die irgendein Antidepressivum erhalten, unterscheiden sich nicht von der medikamentös nicht versorgten Vergleichsgruppe $(\mathrm{t}(61)=1,33, \mathrm{p}=0,187)$. Patienten, die Psychopharmaka erhalten, unterscheiden sich ebenfalls nicht von der nicht medikamentös versorgten Vergleichsgruppe $(\mathrm{t}(52)=1,59, \mathrm{p}=0,118)$.

\section{Therapeutenstudie}

Werden sexuelle Dysfunktionen erkannt? Die Therapeuteneinschätzungen (Tab. 3) verdeutlichen, dass Behandler nicht eindeutig zwischen störungsfreien und Indexpatienten trennen können $\left(\chi^{2}(2)=1,8, p=0,400\right)$, obwohl immerhin $68 \%$ der Therapeuten Einsicht in den Fragebogen genommen haben. Bei fast jedem zweiten Indexpatienten wurde die vermutlich vorliegende sexuelle Dysfunktion nicht erkannt. Bei weiteren $27 \%$ der Indexpatienten war sich der Therapeut unklar darüber, ob eine sexuelle Dysfunktion vorliegt. Darüber hinaus können nur 20\% der Therapeuten, die eine richtige Zuordnung treffen, die Diagnose näher beschreiben.

Werden sexuelle Dysfunktionen thematisiert? Wie in Tabelle 4 ersichtlich, fragen Therapeuten in nur jedem zweiten Fall $(48,3 \%)$ von sich aus nach sexuellen Dysfunktionen. Therapeuten explorieren bei Indexpatienten nicht häufiger als bei Normalpatienten $\left(\chi^{2}(1)=0,7, p=0,796\right)$. Nur jeder vierte Indexpatient (23\%) spricht sexuelle Dysfunktionen selbst an.

Werden sexuelle Dysfunktionen behandelt? Nach Angaben der Therapeuten lagen 12 Fälle sexueller Dysfunktionen vor, davon wurden nur 4 behandelt, 8 nicht. Dabei fühlten sich die befragten Therapeuten im Mittel «gar nicht bis wenig» kompetent, bei der Behandlung sexueller Dysfunktionen. Des Weiteren schätzen Behandler die Bedeutung sexueller Dysfunktionen für die Behandlung anderer Störungen durchschnittlich als lediglich «mittelmäßig» ein. Als Hauptgründe für eine Nichtbehandlung wurden angegeben, dass sie von 
Tab. 3. Beurteilen Therapeuten bei je 30 Patienten mit bzw. ohne sexuelle Dysfunktion das Vorliegen einer sexuellen Beeinträchtigung richtig?

\begin{tabular}{|c|c|c|c|c|}
\hline & \multicolumn{4}{|c|}{ Therapeutenurteil, n (\%) } \\
\hline & $\begin{array}{l}\text { keine sexuelle } \\
\text { Dysfunktion }\end{array}$ & unklar & $\begin{array}{l}\text { sexuelle } \\
\text { Dysfunktion }\end{array}$ & gesamt \\
\hline Patienten ohne sex. Dysfunktion & $18(60,0)$ & $8(26,7)$ & $4(13,3)$ & 30 \\
\hline Indexpatienten & $14(46,6)$ & $8(26,7)$ & $8(26,7)$ & 30 \\
\hline Gesamt & 32 & 16 & 12 & 60 \\
\hline
\end{tabular}

\begin{tabular}{|c|c|c|c|}
\hline & \multicolumn{3}{|c|}{$\begin{array}{l}\text { Wurde die sexuelle Dysfunktion vom Therapeuten } \\
\text { exploriert? n (\%) }\end{array}$} \\
\hline & nein & ja & gesamt \\
\hline \multirow{5}{*}{$\begin{array}{l}\text { Patienten ohne sex. Dysfunktion } \\
\text { Indexpatienten } \\
\text { Gesamt }\end{array}$} & $16(53,3)$ & $14(46,7)$ & 30 \\
\hline & $15(50,0)$ & $15(50,0)$ & 30 \\
\hline & 31 & 29 & 60 \\
\hline & \multicolumn{3}{|c|}{$\begin{array}{l}\text { Wurde die sexuelle Dysfunktion vom Patienten } \\
\text { angesprochen? n (\%) }\end{array}$} \\
\hline & nein & ja & gesamt \\
\hline Patienten ohne sex. Dysfunktion & $30(100)$ & $0(0,0)$ & 30 \\
\hline Indexpatienten & $23(76,7)$ & $7(23,3)$ & 30 \\
\hline Gesamt & 53 & 7 & 60 \\
\hline
\end{tabular}

Tab. 4. Häufigkeit, mit der sexuelle Dysfunktionen thematisiert werden, in Abhängigkeit vom tatsächlichen Vorliegen einer sexuellen Beeinträchtigung
Seiten des Patienten nicht gewünscht wurde $(\mathrm{N}=3)$, dass andere Probleme im Vordergrund standen $(\mathrm{N}=2)$ bzw. dass nur hinsichtlich der Primärdiagnose interveniert wurde $(\mathrm{N}=2)$.

\section{Diskussion und Schlussfolgerungen}

Die vorliegende Studie ging zum einen der Frage nach, wie oft sexuelle Dysfunktionen bei Patienten einer ambulanten Psychotherapieeinrichtung vorkommen. Zum anderen interessierte, ob Psychotherapeuten sexuelle Dysfunktionen adäquat erkennen, mit dem Patienten thematisieren und anschlieBend behandeln.

Die Ergebnisse zur Prävalenz (Studie 1) spiegeln die in anderen Versorgungskontexten gewonnene Datenlage zu Prävalenz und Versorgung sexueller Dysfunktionen wider. Demnach kommen sexuelle Probleme auch im Rahmen ambulanter Psychotherapie anderer psychischer Diagnosen sehr häufig vor. Zugleich bildet sich die weithin angenommene hohe Komorbidität [z.B. Bonierbale et al., 2003; Ernst et al., 1993] sexueller Dysfunktionen mit anderen psychischen Störungen auch in unseren Daten ab. Wir haben sexuelle Beeinträchtigungen sowohl bei Frauen als auch bei Männern beobachtet, wenn auch bei letzteren in geringerem Ausmaß. Die Hälfte der Frauen in ambulanter Psychotherapie beklagt reduziertes oder fehlendes sexuelles Interesse, reduzierte oder fehlende Erregungsfähigkeit, eingeschränkte Orgasmusfähigkeit oder beeinträchtigte Zufriedenheit mit ihrer Sexualität im letzten
Monat, jede dritte Frau äußert eingeschränkte Lubrikationsfunktion. Auf der anderen Seite hat ein Viertel der Männer der Stichprobe im letzten Monat deutlich geringeres sexuelles Interesse und Erregungsfähigkeit, geringere Orgasmusfähigkeit und eingeschränkte Erektionsfähigkeit bei sich beobachtet. Über ein Drittel der Männer äußert generelle Unzufriedenheit mit der eigenen Sexualität im letzten Monat.

Bedauerlicherweise werden vermutliche sexuelle Dysfunktionen jedoch in der Hälfte der Fälle von den Therapeuten nicht erkannt und nicht thematisiert und in der Mehrzahl der Fälle nicht behandelt: Obwohl bei zwei Dritteln der Patienten Einblick in den KFSP genommen wurde, wird die sexuelle Dysfunktion bei der Hälfte der Patienten nicht festgestellt. Die Tatsache, dass nur 25\% der Patienten das Thema Sexualität selbst ansprechen, fordert vom Therapeuten zweifellos mehr Initiative. Jedoch erfolgt diese nur in der Hälfte aller Fälle. Darüber hinaus wird nur jede dritte sexuelle Dysfunktion behandelt, was von den Therapeuten unter anderem mit mangelndem Kompetenzgefühl oder einer vermeintlich geringen Bedeutung der Sexualproblematik begründet wird. Einschränkend ist allerdings zu sagen, dass wir keine Kenntnis über mögliche medizinische oder altersbedingte Ursachen der Dysfunktionen haben und daher die Indikation für deren psychotherapeutische (Mit-)Behandlung nicht sicher beurteilen können.

Die defizitäre Versorgung sexueller Dysfunktionen auch im ambulant-psychotherapeutischen Setting verlangt dringend Veränderungen in der diagnostischen und therapeutischen 
Ausbildung von Psychotherapeuten. Angesichts der Tatsache, dass Sexualität und sexuelle Dysfunktionen in der Ausbildung zum psychologischen Verhaltenstherapeuten bisher nur eine rudimentäre Rolle spielen und umfangreiche empirische Evidenz dafür besteht, dass Behandler, von denen eigentlich ein offener Umgang mit dem Thema Sexualität erwartet wird, große Hemmungen im Thematisieren sexueller Probleme zeigen [z.B. Meerabeau, 1999], stellt sich die Diskussion einer stärkeren Verankerung des Themas Sexualität in den Curricula der Aus- und Weiterbildung. Durch eine bessere Integration sexueller Funktionsstörungen in das allgemeine Diagnostik- und Interventionsrepertoire von Psychotherapeuten, das Bewusstmachen eigener Schwellen im Umgang mit diesem Thema und eine damit verbundene Enttabuisierung könnten psychotherapeutische Ambulanzen adäquate, bedarfsgerechte Einrichtungen für diesen bisher vernachlässigten Versorgungsbereich werden.

Die Vernachlässigung des Themas mag jedoch nicht nur mit den Schwierigkeiten zu erklären sein, sexuelle Probleme adäquat anzusprechen und zu behandeln. Vielleicht werden sexuelle Störungen auch als wenig beeinträchtigend angesehen. Schließlich kommt es wegen sexueller Störungen und Probleme selten zu Krankschreibungen, und die Bereitschaft der Krankenkassen zur Kostenübernahme wird gering sein. Andererseits aber gilt: Fortbestehende psychische Störungen und Probleme sind allgemein ein zentraler Risikofaktor für psychosoziale Einschränkungen und Folgeprobleme. Unbehandelte sexuelle Störungen dürften zumindest langfristig Paarprobleme und affektive Störungen vorhersagen. Die Verursachung von Leiden bzw. zwischenmenschlichen Schwierigkeiten ist wesentlicher Teil der DSM-IV-Definition von sexuellen Funktionsstörungen. Und es ist weithin bekannt, dass Unzufriedenheit in der Beziehung negative Auswirkungen auf die psychische und physische Gesundheit der Partner hat [Halford et al., 1997]. So steht sie unter anderem im Zusammenhang mit Depressionen [Floyd und Zmich, 1991], Substanzmissbrauch [Halford und Osgarby, 1993; Maisto et al, 1988] und einer erhöhten Infektionsanfälligkeit [KiecoltGlaser et al., 1987].

Für die Praxis scheint es ein sehr einfacher und ökonomischer Weg, mit einem Screening-Verfahren, dessen Bearbeitung keinem unserer Patienten Schwierigkeiten gemacht hat, das Thema Sexualität aufzugreifen und in den diagnostischen Prozess zu integrieren. Wenn das Problem einmal identifiziert ist, könnten erste psychoedukative Informationen auch bereits in der Wartezeit erfolgen [Helbig et al., 2004].
Methodenkritisch ist abschließend anzumerken, dass uns zum Zeitpunkt der Untersuchung nur ein Screening-Maß für sexuelle Dysfunktion zur Verfügung stand, das es nicht erlaubte, Diagnosen einer sexuellen Funktionsstörung zu stellen und zu prüfen. Es ist deshalb noch einmal zu betonen, dass hier lediglich sexuelle Dysfunktionen angesprochen wurden und Aussagen über vorliegende sexuelle Störungen nicht möglich sind. Diagnostisch aussagekräftigere Instrumente, zum Beispiel strukturierte Interviews für sexuelle Funktionsstörungen [SISEX, Reitz et al., 2005], lagen zum Untersuchungszeitpunkt noch nicht vor. Ergänzende Analysen der Übereinstimmung der durch KFSP bzw. SISEX gewonnenen Klassifikation von Patienten ohne sexuelle Dysfunktion vs. Patienten mit Verdacht auf sexuelle Dysfunktion sprechen jedoch für eine zufriedenstellende Zuordnung durch den KFSP.

Darüber hinaus ist die hier untersuchte Therapeutenstichprobe nur begrenzt repräsentativ, und es kann nicht eindeutig geklärt werden, wie weitreichend und allgemeingültig therapeutische Zurückhaltung und mangelndes Kompetenzerleben im Umgang mit dem Thema Sexualität tatsächlich sind. Unsere Ergebnisse sagen wenig darüber aus, wie andere, insbesondere erfahrenere oder an anderen Instituten ausgebildete Verhaltenstherapeuten das Problem handhaben. Vermutlich kann Erfahrung (auch Lebenserfahrung) dazu beitragen, das Thema Sexualität in der diagnostischen Phase der Therapie angemessen anzusprechen. Außerdem ist für einen kompetenten Umgang mit der meist komorbiden Problematik, die in der Regel nicht den Konsultationsgrund darstellt, ein geeigneter Kurs zu sexuellen Funktionsstörungen [Vogelgesang, 1997; vgl. Hoyer, 2005] als Teil der vertiefenden Ausbildung eine wichtige Voraussetzung. Nur wenn beide Faktoren - langjährige Erfahrung und spezifische Ausbildung - nicht gegeben sind, ist aus unserer Sicht mit ähnlichen Ergebnissen wie in unserer Studie zu rechnen. Angesichts der eingangs zitierten Untersuchungen vermuten wir jedoch, dass die beschriebenen Versorgungsdefizite sich nicht auf die untersuchte Ambulanz beschränken. Wir möchten deshalb nicht nur zu Ergänzungen in der Aus- und Weiterbildung von Psychotherapeuten, sondern auch zu umfassenderer empirischer Erfassung der realen Versorgungssituation im Bereich sexueller Dysfunktionen und sexueller Funktionsstörungen ermutigen.

\section{Dank}

Wir danken allen beteiligten Patienten und Therapeuten für die Unterstützung und uneigennützige Mitarbeit an der Studie.

\section{Literatur}

Bonierbale M, Lancon C, Tignol J: The ELIXIR study: evaluation of sexual dysfunction in 4557 depressed patients in France. Curr Med Res Opin 2003;19(2): 114-124

Dunn KM, Croft PR, Hackett GI: Sexual problems: a study of the prevalence and need for health care in the general population. Fam Pract 1998;15(9):519-524.
Ernst C, Foldenyi M, Angst J: The Zurich Study. Sexual dysfunction and disturbances in young adults - data of a longitudinal epidemiologic-study. Eur Arch Psych Clin Neurosci 1993;243(3-4):179-188.

Fahrner EM: Psychologische Behandlung von Sexualstörungen bei männlichen Alkoholabhängigen. München, Röttger, 1985.
Floyd FJ, Zmich DE: Marriage and the parenting partnership: perceptions and interactions of parents with mentally retarded and typically developing children. Child Dev 1991;62:1434-1448.

Halford WK, Kelly A, Markman HJ: The concept of a healthy marriage; in Halford WK, Markman HJ (eds) Clinical Handbook of Marriage and Couples Intervention. Chichester, Wiley, 1997, pp 3-12. 
Halford WK, Osgarby S: Alcohol abuse in clients presenting with marital problems. J Fam Psych 1993;6: $1-11$.

Helbig S, Hähnel A, Weigel B, Hoyer J: Wartezeiten und wie sie zu nutzen sind. Verhaltenstherapie 2004; 14:294-302.

Hoyer J: Zur theoretisch-methodischen Ausbildung in Verhaltenstherapie; in Laireiter AR, Willutzki U (Hrsg): Ausbildung in Verhaltenstherapie. Göttingen, Hogrefe, 2005, pp 201-221.

Hoyer J, Beauducel A, Franke GH: Kriterien der Angstdiagnostik - woran der Anwender auch denken muss, in Hoyer J, Margraf J (Hrsg): Angstdiagnostik Grundlagen und Testverfahren. Heidelberg, Springer, 2003, pp 77-92.

Johnson SD, Phelps DL, Cottler LB: The association of sexual dysfunction and substance use among a community epidemiologic sample. Arch Sex Behav 2004;33 (1):55-63.

Kiecolt-Glaser JK, Fisher LD, Ogrocki BS, Stout JC, Speicher CE, Glaser R: Marital quality, marital disruption and immune function. Psych Med 1987;49:13-33.

Kockott G, Pfeiffer W: Sexual disorders in nonacute psychiatric outpatients. Compr Psychiatry 1996;37(1): 56-61.
Kommer D, Lambert M, Moehr I, Trierweiler A, Zielke M: Zur Prävalenz sexueller Funktionsstörungen bei Patienten, die wegen anderweitiger psychosomatischer oder psychischer Störungen stationär behandelt werden; in Zielke M, Mark N (Hrsg): Fortschritte der angewandten Verhaltensmedizin. Konzeption, Grundlagen, Therapie, Evaluation, vol 1. Berlin, Springer, 1990, pp 147-167.

Labbate LA, Lare SB: Sexual dysfunction in male psychiatric outpatients: validity of the Massachusetts General Hospital Sexual Functioning Questionnaire. Psychother Psychosom 2001;70:221-225.

Laumann E, Paik A, Rosen R: Sexual dysfunction in the United States: prevalence and predictors. J Am Med Assoc 1999;281:537-544.

Maisto SA, O'Farrell TJ, Connors GJ, McKay JR, Pelcovits M: Alcoholics' attributions of factors affecting their relapse to drinking and reasons for terminating relapse episodes. Addict Behav 1988;13:79-82.

Meerabeau L: The management of embarrassment and sexuality in health care. J Adv Nurs 1999;29(6): 1507-1513.

Moynihan R: The making of a disease: female sexual dysfunction. Br Med J 2003;326:45-47.
Nicolosi A, Laumann EO, Glasser DB, Moreira ED Jr, Paik A, Gingell C: Sexual behavior and sexual dysfunctions after age 40: the global study of sexual attitudes and behaviors. Urology 2004;64(5):991-997.

Nusbaum MRH, Singh AR, Pyles AA: Sexual healthcare needs of women aged 65 and older. J Am Geriatr Soc 2004;52(1):117-122.

O'Farrell TJ, Choquette KA, Cutter HSG, Birchler GR: Sexual satisfaction and dysfunction in marriages of male alcoholics: Comparison with non-alcoholic martially conflicted and non-conflicted couples. J Stud Alcohol 1997;58:91-99.

Read S, King M, Watson J: Sexual dysfunction in primary medical care: prevalence, characteristics and detection by the general practitioner. J Public Health Med 1997;19(4):387-391.

Reitz D, Küpper B, Hoyer J: Strukturiertes Interview für sexuelle Funktionsstörungen - erste Ergebnisse. Psychother Psychosom Med Psychol 2005;55:147.

Vogelgesang M: Modell eines psychosexualtherapeutischen Seminars in der verhaltenstherapeutischen Weiterbildung. Verhaltensther Verhaltensmed 1997;18: 317-341.

Wittchen HU, Pfister H (Hrsg): DIA-X Interviews: Manual für Screeningverfahren und Interview: PCProgramm zur Durchführung des Interviews (Längsund Querschnittuntersuchung), Auswertungsprogramm. Frankfurt, Swets, 1997. 